\title{
LTR promoter activity of SRLV genotype E, strain Roccaverano
}

\author{
M. Juganaru • R. Reina • E. Grego • M. Profiti • \\ S. Rosati
}

Published online: 18 May 2010

(C) Springer Science+Business Media B.V. 2010

\begin{abstract}
The highly divergent, small ruminant lentivirus (SRLV) genotype E Roccaverano strain has a full genome consisting of 8,418 nucleotides, which lack the entire dUTPase subunit of the pol gene, the $v p r$-like accessory gene, and the 71-bp repeat of the U3 region within the long terminal repeat (LTR). These deletions affect in reverse transcriptase fidelity in non-dividing cells (dUTPase and vpr-like) and in the regulation of viral replication. Surprisingly, this SRLV strain was able to replicate efficiently in non-dividing cells (i.e., blood-derived macrophages), while replication in fibroblastic-like cells was somewhat restricted. To evaluate whether this observation was due to the presence/absence of specific transcription factors within these fibroblasts, U3 transcriptional activity was measured in the different cell types and revealed that both fibroblasts and macrophages were able to activate the viral promoter in the same manner. Among the transcription factor-binding sites present within the U3 region, the highly conserved Ap4 tandem repeat was shown to be sufficient for LTR promoter activity.
\end{abstract}

Keywords Small ruminant lentivirus $\cdot$ Genotype E $\cdot$ LTR

$\begin{array}{ll}\text { Abbreviations } \\ \text { SRLV } & \text { small ruminant lentivirus } \\ \text { MVV } & \text { Maedi Visna virus } \\ \text { CAEV } & \text { caprine arthritis encephalitis virus } \\ \text { LTR } & \text { long terminal repeat } \\ \text { SM } & \text { synovial membrane } \\ \text { CP } & \text { choroid plexus } \\ \text { LC } & \text { lung } \\ \text { SP } & \text { spleen }\end{array}$

M. Juganaru $(\bowtie) \cdot$ R. Reina $\cdot$ E. Grego $\cdot$ M. Profiti $\cdot$ S. Rosati

Dipartimento di Produzioni Animali, Epidemiologia ed Ecologia, Università di Torino, Torino, Italy

e-mail: mariamagdalena.juganaru@unito.it 


\section{Introduction}

Recently, a new genotype E among small ruminant lentiviruses (SRLVs), showing deletions corresponding to the dUTPase subunit of the pol gene and vpr-like gene involved in reverse transcriptase (RT) fidelity in non-diving cells and the 71-bp repeat of the long terminal repeat (LTR), was described. MVV (Maedi Visna virus) replication is controlled primarily at the level of transcription, which is, in turn, regulated by the bond between cis-acting DNA elements located in the viral LTRs by cellular transcription factors (Barros et al. 2005). Basal activity of the SRLV promoter is much higher than that of human immunodeficiency virus-1 and does not require the trans-activating protein Tat. Indeed, the caprine arthritis encephalitis virus (CAEV) Tat protein has been proposed as a vpr-like protein due to its lack of trans-activating activity. LTRs have been described as viral transcription promoters and their U3 region as an enhancer where transcription factorbinding sites are present. Several studies have investigated the relative role of each individual site in enhancing replication and tropism (Oskarsson et al. 2007; Murphy et al. 2007). Several SRLV strains characterized so far contain repeats within U3 that increase downstream transcription and increased virulence. Actually, it has been demonstrated that deletion of a CAAAT sequence from either one of the repeats results in poor virus growth in sheep choroid plexus cells, suggesting that U3 duplication is associated with neurovirulence (Oskarsson et al. 2007). On the other hand, MVV-AML-defective chimeric viruses showed both lower replication and promoter activity due to the absence of the U3 repeat. Both the presence of cell-specific transcription factors that bind the viral U3 region and the importance of the highly conserved AP4 site have also been proposed to play roles in virulence (Barros et al. 2005).

In this report, we have characterized the LTR promoter activity of the Roccaverano strain in vitro and studied the relative role of the different enhancer-binding sites found in the U3 region. Genotype E LTR promoter activity was high in all cells tested, reaching values comparable to the CAEV B1 subtype as a positive control. Among the sites found within U3, deletion of AP4 and an AP4-like site reduced promoter activity significantly with poor influence of AML, GAS, TAS, and CAAAT sites.

\section{Materials and methods}

Virus and Cells The genotype E Roccaverano strain was previously isolated and sequenced (Reina et al. 2009). CAEV field isolate TO1/89 was originally obtained by synovial membrane explants from an arthritic goat and partially characterized in a previous study (Grego et al. 2002). This field isolate was used as a conventional B1 subtype strain. Primary cell cultures from caprine fetal synovial membrane (SM), choroid plexus (CP), lung (LC), and spleen (SP) were established using MEM (Sigma) supplemented with 2\% L-glutamine, $10 \%$ fetal bovine serum, and antibiotics/antimycotics (Life Tech). Blood-derived macrophages (BDMs) were obtained from uninfected goats belonging to a long-term SRLV-free flock, after 9-day culture on a Teflon surface to allow differentiation. The cell population was identified as having a monocyte/macrophage lineage by morphological evaluation, strong adhesion to a plastic surface, and immunocytochemistry using the Mab VPM32.

Plasmids construction The U3 region, covering the transcription factor-binding site region, was amplified from infected cultures using standard PCR protocols with specific primers possessing MluI and XhoI sites (Roccaverano GenBank EU293537) (Fig. 1). The PCR 


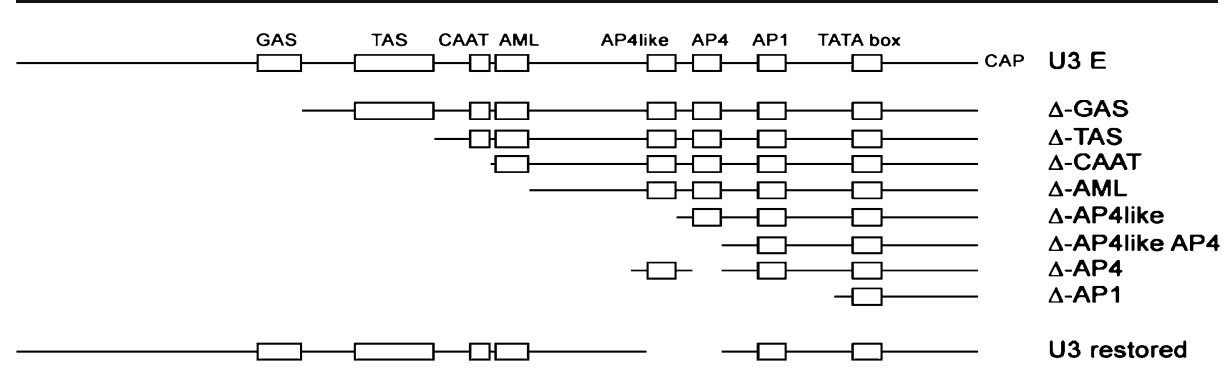

Fig. 1 U3 constructs made by deleting different enhancer-binding sites one at a time. Transcription factorbinding sites are indicated by white boxes, and nucleotide deletions are indicated by lines between boxes

products were cloned into a pCAT-Basic vector (Promega) after MluI/XhoI digestion. The same region corresponding to $\mathrm{CAEV}-\mathrm{TO} / 89$ was cloned following the same protocol and used as a positive control. The pCAT-Basic vector contains chloramphenicol acetyltransferase (CAT) as a reporter gene and lacks eukaryotic promoter and enhancer sequences, allowing maximum flexibility in cloning putative regulatory sequences. We used as a second positive control the pCAT-Control vector, which contains SV40 promoter and enhancer sequences to which the relative expression of each construct was based. After the first set of transfection experiments, eight additional constructs were generated as reported in Fig. 1. Inserts were amplified by PCR or synthetically generated, based on their lengths. A final construct was generated to restore all enhancer elements except the AP4 tandem repeat using primers possessing Acc65I and MluI restriction sites. The sequence present within each construct was verified using an automated DNA Sequencer (ABI PRISM 310 Genetic Analyzer). All plasmids were propagated in E. coli TOP 10 and purified using an endotoxin-free miniprep kit (Qiagen).

Transfection experiments Plasmid vectors described above were used in transfection experiments. Promoter activity was estimated by quantifying CAT production using an ELISA procedure (Roche). Transfection was carried out in 24-well plates using $0.9 \mu \mathrm{g}$ of the aforementioned plasmids and Lipofectamine 2000 (Invitrogen) at a 1:1 ratio ( $\mu$ g DNA/ $\mu \mathrm{L}$ Lipofectamine) in the case of SM, CP, SP, and LC cells. BDM cell transfection was performed using polyethylenimine microparticles (PEI) conjugated with mannose that uses the ability of macrophages to internalize the complex via the mannose receptor at a $1: 10$ ratio (DNA:PEI). In both cases, cells were lysed $48 \mathrm{~h}$ after transfection and processed as specified to quantify CAT production (Calbiochem). Transfection efficiency was evaluated in situ, using a $\beta$-galactosidase expression staining kit (Stratagene).

\section{Results}

The U3 region flanking the most important enhancer elements from Roccaverano and CAEV-TO/89 strains was cloned into pCAT and used to transfect SM, PC, LC, SP, and BDM cells. There were no differences between transcriptional activities among CAEV-TO/ 89, Roccaverano, and SV40 promoters (data not shown). Additional constructs were made by deleting the GAS, TAS, CAAAT, AML, AP4, AP4-like, and AP1 enhancer elements of U3 one at a time (Fig. 1) and used to transfect SM cells. Deleting singly in this order (GAS, TAS, CAAAT, and AML), transcriptional activity did not decrease. However, deletion of AP4 resulted in a significant decrease in CAT expression. Additionally, we found that 
deletion of an AP4-like site (sequence CTGCTGA) also resulted in lower LTR activity. The $\Delta$-AP1 construct consisting of the U3 region with only the spacer region above the TATA box did not show any activity, comparable with the negative control. Finally, a construct including the aforementioned elements but without the AP4 tandem repeat showed high transcriptional activity compared with the entire U3 regions of both Roccaverano and SV40 promoters (Fig. 2).

\section{Discussion}

In this study, we characterized the LTR promoter activity of the Roccaverano strain belonging to SRLV genotype $\mathrm{E}$ in different caprine cell types to clarify the specific role of each element in terms of promoter activity. For both MVV and CAEV, the presence of a 35or 71-nt repeat that duplicates the transcription enhancer elements within the U3 region has been described. Recently, two cytokines, TNF- $\alpha$ and IFN- $\gamma$, have been demonstrated to activate the CAEV promoter through mechanisms involving this repeat region, thereby increasing viral load, tissue distribution, and lesion severity. Other transcription enhancer elements such as AP1, AML, or CAAAT sites were decreased in the absence of this repeat. Thus, based on current knowledge, the deletion corresponding to the 71-bp repeat of the LTR U3 region present in genotype E confers lower promoter activity. In fact, LTR from strain P1OLv (genotype A) lacking the repeat conferred a slow/low phenotype to the highly pathogenic molecular clone K1514 (Barros et al. 2005).

However, the Roccaverano LTR was as active as SV40, suggesting that the impaired viral production seen in previous studies was unlikely due to the absence of transcription factors using caprine cells. Moreover, LTR trancriptional activity was similar in SM, CP, LC, SP, and BDM, excluding the differential production of DNA-binding proteins specific to cell type. Rather it could be explained if few viruses were able to enter into these cells because of a lower entry ratio.

Detailed characterization of the single binding sites revealed that only AP4 sites are essential for enhanced LTR promoter activity. Interestingly, AP4 sites are highly conserved among SRLV strains belonging to different genotypes. However, the construct, including all enhancer elements except the AP4 tandem repeat, showed high LTR activity. This could be likely due to the relative nucleotide distance between these elements and the TATA box, mimicking AP4 location.

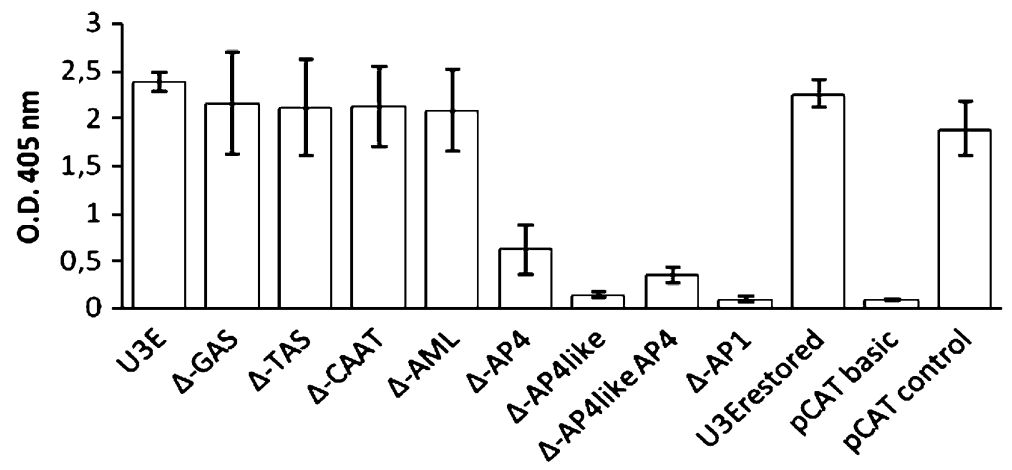

Fig. 2 Promoter activity of the Roccaverano strain U3 region and other constructs after deletion of sites 
This study demonstrates poor association between lentivirus-induced pathology and LTR promoter activity, at least, in this low pathogenic caprine lentivirus, providing indirect evidence that cell tropism may be mediated by the receptor-binding site, rather than transcription factors. In our model, the tandem repeat of the AP4 site seemed sufficient for normal LTR activity.

Acknowledgments We are grateful to Paolo Provero for critical review.

\section{References}

Barros SC, Andrésdóttir V, Fevereiro M (2005) Cellular specificity and replication rate of Maedi Visna virus in vitro can be controlled by LTR sequences. Arch Virol 150(2):201-213

Grego E, Profiti M, Giammarioli M, Giannino L, Rutili D, Woodall C, Rosati S (2002) Genetic heterogeneity of small ruminant lentiviruses involves immunodominant epitope of capsid antigen and affects sensitivity of single-strain-based immunoassay. Clin Diagn Lab Immunol 9(4):828-832

Murphy B, Jasmer DP, White SN, Knowles D (2007) Localization of a TNF-activated transcription site and interactions with the gamma activated site within the CAEV U3 70 base pair repeat. Virology 364 (1):196-207

Oskarsson T, Hreggvidsdóttir HS, Agnarsdóttir G, Matthíasdóttir S, Ogmundsdóttir MH, Jónsson SR, Georgsson G, Ingvarsson S, Andrésson OS, Andrésdóttir V (2007) Duplicated sequence motif in the long terminal repeat of Maedi Visna virus extends cell tropism and is associated with neurovirulence. J Virol 81(8):4052-4057

Reina R, Grego E, Bertolotti L, De Meneghi D, Rosati S (2009) Genome analysis of small-ruminant lentivirus genotype E: A caprine lentivirus with natural deletions of the dUTPase subunit, vpr-like accessory gene, and 70-base-pair repeat of the U3 region. J Virol 83(2):1152-1155 